\title{
Experimental Test and Analysis of AC Losses in Multifilamentary $\mathrm{MgB}_{2}$ Wire
}

\author{
Jiawen Xi, Xiaoze Pei, Jie Sheng, Hideki Tanaka, Yota Ichiki, Min Zhang, and Weijia Yuan
}

\begin{abstract}
AC losses in superconductors are essential for the design of cooling system for large scale power applications. Magnesium diboride $\left(\mathrm{MgB}_{2}\right)$ superconducting wires have been investigated and manufactured over the last decade due to cheap raw materials and flexibility for coil design. In addition, multifilamentary $\mathrm{MgB}_{2}$ wires have been manufactured to reduce $\mathrm{AC}$ losses. In this paper, self-field $\mathrm{AC}$ losses of multifilamentary $\mathrm{MgB}_{2}$ wires with magnetic barrier were investigated using both experimental and numerical methods. A short straight wire sample and a coil sample were tested under various temperatures and frequencies between $16 \mathrm{~Hz}$ and $128 \mathrm{~Hz}$. The test results show that the transportation loss is independent of the operating temperature. On basis of both theoretical and numerical study, it is found that hysteresis loss in superconductor accounts only for a small fraction of the transportation losses, ferromagnetic hysteresis loss in the magnetic barrier dominates when the transport current is low, whereas eddy current loss dominates when the transport current is close to the critical current.
\end{abstract}

Index Terms - AC losses, eddy current loss, ferromagnetic loss, hysteresis loss, magnesium diboride, multifilamentary superconductor.

\section{INTRODUCTION}

$\mathbf{T}$ RANSPORT current AC losses include hysteresis loss in superconducting material, eddy current loss in normal metal parts of the superconducting wire, and ferromagnetic hysteresis loss in magnetic materials [1]-[5]. This can determine the design of cooling system for large scale application in power system. In addition, the superconductor may lose superconductivity and current carrying capability if suffering from excess AC loss. Reducing AC losses can cut down cooling cost considerably for practical application [6], [7]. Therefore, it is worthwhile to study and understand AC losses behavior in superconductors.

Magnesium diboride $\left(\mathrm{MgB}_{2}\right)$ was discovered to show superconductivity below $39 \mathrm{~K}$ in 2001 in Japan [8]. Because of inexpensive raw materials, relatively simple manufacturing process and versatile shapes, $\mathrm{MgB}_{2}$ superconducting wires

Corresponding author: Xiaoze Pei

This work was funded as part of the UK EPSRC, Developing Superconducting Fault Current Limiters (SFCLs) for Distributed Electric Propulsion Aircraft: EP/S000720/1.

J. Xi and X. Pei are with the Department of Electronic and Electrical Engineering, The University of Bath, Bath BA2 7AY, U.K. (e-mail: j.xi@bath.ac.uk; x.pei@bath.ac.uk).

J. Sheng is with the Department of Electronic Information and Electrical Engineering, Shanghai Jiao Tong University, Shanghai, China (e-mail: sj1@sjtu.edu.cn).

H. Tanaka and Y. Ichiki are with the Hitachi Research Laboratory, Hitachi, Ltd., Ibaraki, 319-1292, Japan.

M. Zhang, W. Yuan are with the Department of Electronic and Electrical Engineering, University of Strathclyde, Glasgow, G1 1XW, UK.

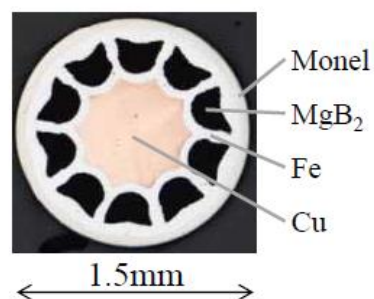

Fig. 1. Cross-sectional view of $\mathrm{MgB}_{2}$ wire.

have attracted wide research interests and have great potential in practical applications, such as superconducting fault current limiters (SFCL), superconducting cables and superconducting machines [6]. Multifilamentary $\mathrm{MgB}_{2}$ wires have been manufactured to reduce AC losses [9], [10]. Kajikawa et al. [11] have studied transport current $\mathrm{AC}$ loss of a single core $\mathrm{MgB}_{2}$ wire with copper sheath, they found the dependence between $\mathrm{AC}$ loss and frequency might be caused by the copper sheath. Hong et al. [12] found that $\mathrm{AC}$ loss in multifilamentary $\mathrm{MgB}_{2}$ wire was lower than monofilamentary wire with the same current. Young et al. [13] investigated multifilamentary $\mathrm{MgB}_{2}$ wires with ferromagnetic nickel $(\mathrm{Ni})$ sheath and weakly magnetic nickel-chromium $(\mathrm{NiCr})$ sheath, the wire with $\mathrm{Ni}$ sheath produces higher AC losses.

This paper investigates self-field AC losses of a new multifilamentary $\mathrm{MgB}_{2}$ wire with magnetic barrier manufactured by Hitachi Ltd. According to Norris model [14], hysteresis losses can be reduced by filamentation of wire. However, total AC losses may be increased due to the existence of eddy current loss and ferromagnetic hysteresis loss. It is worthwhile to investigate $\mathrm{AC}$ losses of the multifilamentary $\mathrm{MgB}_{2}$ wire. $\mathrm{AC}$ losses of a straight wire sample and a coil sample were measured under various temperatures, supply frequencies and transport current values. Numerical modelling of hysteresis loss and eddy current loss of the straight wire sample was performed using finite element (FE) models. The behavior of $\mathrm{AC}$ losses in multifilamentary $\mathrm{MgB}_{2}$ wire is analyzed and discussed based on experimental and modelling results. Some suggestions on improving the manufacture of the conductor are also provided.

\section{EXPERIMENTAL SETUP}

\section{A. $\mathrm{MgB}_{2}$ Sample Preparation}

Multifilamentary $\mathrm{MgB}_{2}$ wire manufactured by Hitachi Ltd. using in-situ power in tube (PIT) method was investigated [15], [16]. Fig. 1 presents the cross-sectional view of $\mathrm{MgB}_{2}$ 
(a)

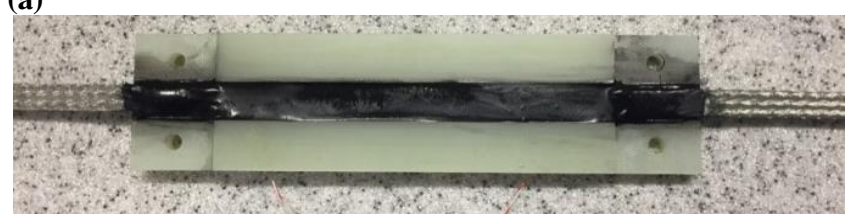

(b)

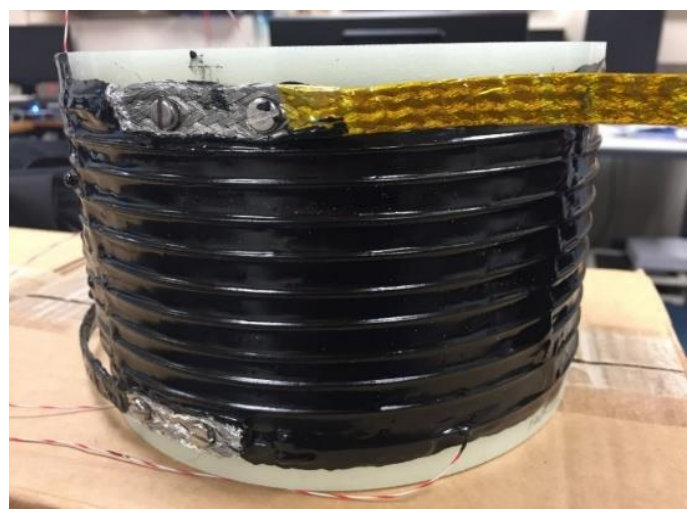

Fig. 2. (a) Short straight wire sample placed into the groove of a G10 board, (b) Coil sample wound onto a G10 tube.

round wire. The diameter of the wire is $1.5 \mathrm{~mm}$. The wire has $10 \mathrm{MgB}_{2}$ filaments surrounded by iron barrier. The copper matrix is used to enhance the thermal stability. The iron barrier prevents the reaction between copper and magnesium. The wire utilizes Monel sheath to improve its mechanical strength.

A short straight wire and a coil were investigated here in this paper. The 190-mm-long short straight wire sample is presented in Fig. 2 (a). Copper braids were soldered onto both ends of the straight wire, and two voltage taps were soldered with $100 \mathrm{~mm}$ apart. The wire with copper braids was placed into the groove of a fiberglass G10 board. The groove was then filled with epoxy resin to improve the thermal conductivity and mechanical strength.

The $\mathrm{MgB}_{2}$ coil was initially wounded on a metal bobbin for heat treatment. As shown in Fig. 2 (b), the coil was then wound onto a G10 tube for testing. The G10 tube had the same diameter as the metal bobbin and the distance between each turn of the coil was maintained the same to minimize the coil deformation. Two coil terminals were soldered on the copper bars. The coil had 10 turns with a diameter of $179 \mathrm{~mm}$, the total length of the wire was about $5.6 \mathrm{~m}$. Two voltage taps were soldered on the $\mathrm{MgB}_{2}$ coil. The epoxy resin was finally coated onto the coil to hold it in place.

The sample was assembled into a cryostat system for testing. The temperature of the cold head in the cryostat can be controlled from $17 \mathrm{~K}$ to $80 \mathrm{~K}$. A temperature sensor was attached to the G10 surface to measure the real-time temperature of the sample during testing.

\section{B. Critical Current Measurement}

The four-point method was used to measure critical current. A DC programmable power supply was controlled by a LabVIEW program to apply increasing current to $\mathrm{MgB}_{2}$ wire. The LabVIEW system can control the rate of current increase, such as $1 \mathrm{~A} / \mathrm{s}, 2 \mathrm{~A} / \mathrm{s}$ and $3 \mathrm{~A} / \mathrm{s}$. The test sample was installed in the cryostat, and then connected in series with the DC power sup-

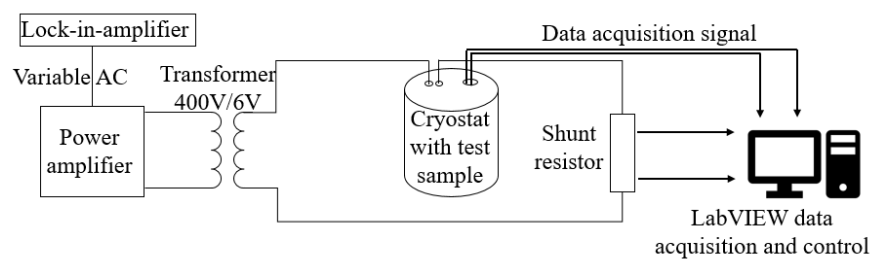

Fig. 3. AC transport current loss measurement schematic.

ply and a shunt resistor. The voltage across the test sample was measured from voltage taps, and the current was measured using the shunt resistor. Both the voltage and current signals were recorded using the LabVIEW data acquisition system. In this paper, the critical current $I_{c}$ is obtained when the voltage across the sample reaches $1 \mu \mathrm{V} / \mathrm{cm}$.

\section{AC Transport Current Loss Measurement}

Two methods for measuring AC losses were compared by Pei [17]: a direct method which measured the current and voltage of the test sample directly; and an indirect method which inserted a cancelling coil to remove the inductive component of the test sample voltage. AC losses measured by two methods were similar for low inductance coil. In this paper, a testing circuit based on the direct method was built to measure self-field AC losses.

The schematic diagram of AC loss measurement is shown in Fig. 3, which includes a lock-in amplifier, a power amplifier, a voltage step-down transformer, a shunt resistor and a LabVIEW data acquisition system. A sinusoidal signal was produced by the lock-in-amplifier and then amplified using the power amplifier. The voltage step-down transformer was used to further increase the current level. The voltage and current signals were measured and recorded by the LabVIEW data acquisition system. The real power representing AC loss can be calculated by integrating the instantaneous power over a cycle:

$$
P=Q / T=\left(\int_{0}^{T} V \times I d t\right) / T
$$

where $P$ and $Q$ represent the AC loss in watt per meter $(\mathrm{W} / \mathrm{m})$ and in joule per meter per cycle $(\mathrm{J} / \mathrm{m} /$ cycle $)$, respectively. $V$ is the instantaneous voltage across the test sample, $I$ is the instantaneous current flowing through the test sample and $T$ is the duration of one cycle.

\section{EXPERIMENTAL RESULTS AND DISCUSSION}

\section{A. Critical Current}

Fig. 4 illustrates the critical current of the short straight wire as a function of temperature from $23 \mathrm{~K}$ to $36 \mathrm{~K}$. It can be seen that the critical current reduces considerably as the temperature increases. The critical current values are $277 \mathrm{~A}$ and $226 \mathrm{~A}$ at $23 \mathrm{~K}$ and $30 \mathrm{~K}$, respectively. In addition, the critical current at $23 \mathrm{~K}$ is almost twice of that at $36 \mathrm{~K}$. 


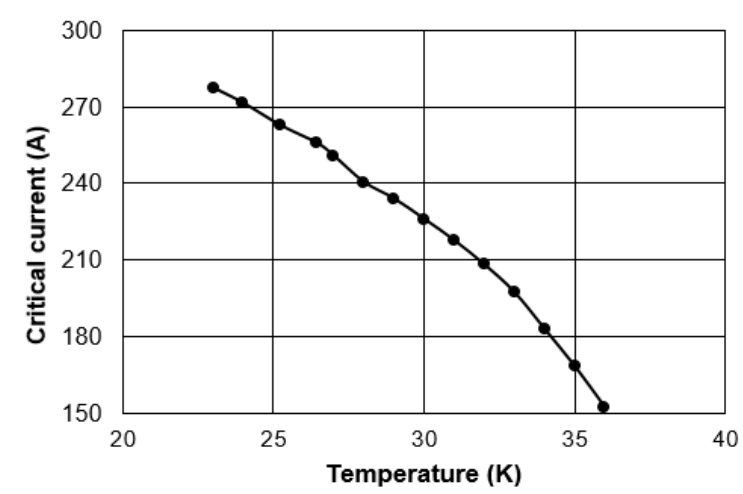

Fig. 4. Critical current of short straight wire from $23 \mathrm{~K}$ to $36 \mathrm{~K}$.

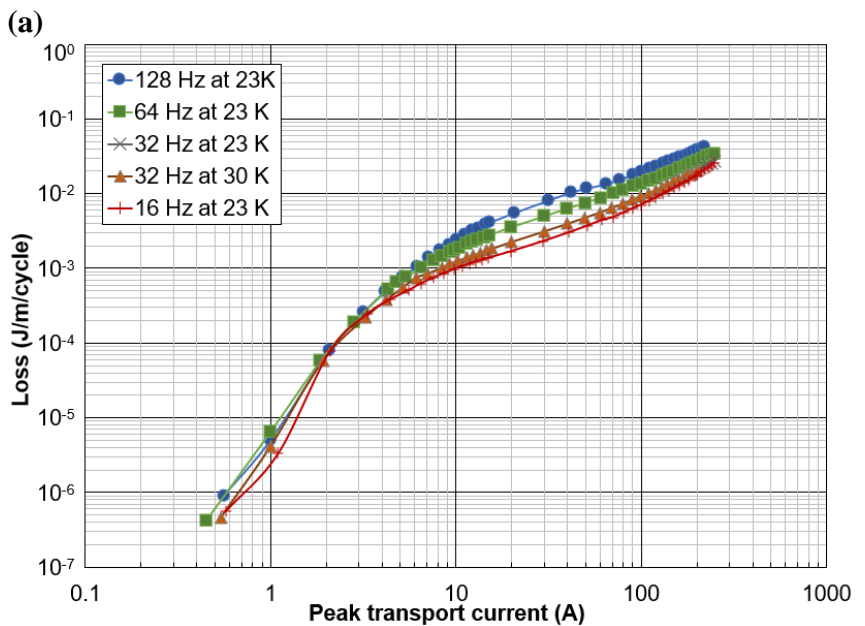

(b)

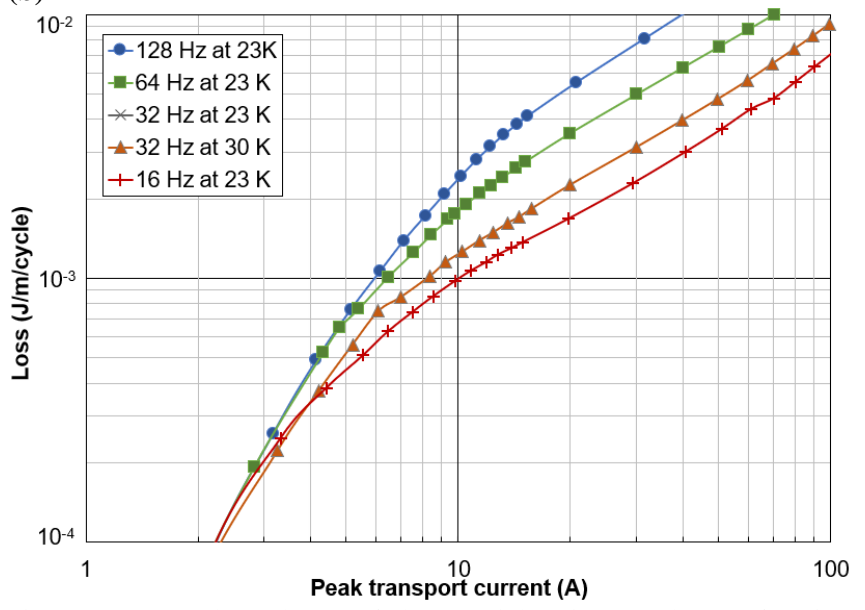

Fig. 5. Measured AC losses of short straight wire sample as a function of peak transport current: (a) full range and (b) zoom at $10 \mathrm{~A}$

\section{B. AC Transport Current Losses}

\section{Straight wire:}

AC losses of the short straight wire were measured at $23 \mathrm{~K}$ with supply frequency from $16 \mathrm{~Hz}$ to $128 \mathrm{~Hz}$. AC losses were also measured at $30 \mathrm{~K}$ with supply frequency of $32 \mathrm{~Hz}$. Fig. 5 shows $\mathrm{AC}$ losses of the short straight wire at the operating temperature of $23 \mathrm{~K}$ and $30 \mathrm{~K}$. It is obvious that the AC loss at the operating temperature of $30 \mathrm{~K}$ is similar to the value at operating temperature of $23 \mathrm{~K}$. It shows that the measured AC losses does not change with operating temperature [13]. Hys-

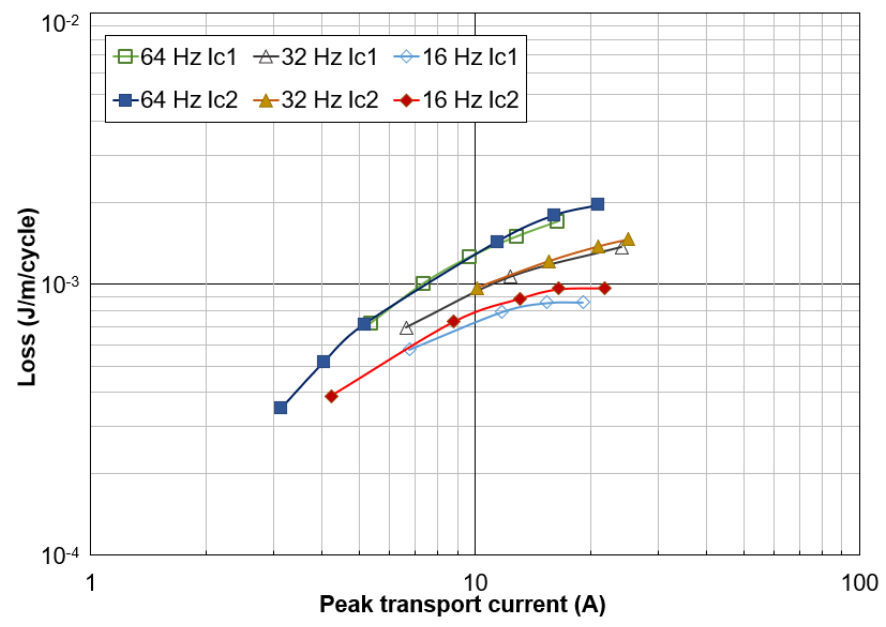

Fig. 6. Measured AC losses of coil sample as a function of peak transport current under two critical currents: Ic1 of $34 \mathrm{~A}$ and Ic2 of $47 \mathrm{~A}$.

teresis loss in superconductor predicted by Norris model changes because the critical current changes with operating temperature [14]. This indicates that hysteresis loss is a small portion of the measured AC losses.

It can also be seen in Fig. 5 that the measured AC losses grow approximately with the cube of the transport current when the current is under $5 \mathrm{~A}$. As the current increases, the gradient of measured AC losses changes sharply and follows almost linearly with current when the current is higher than $5 \mathrm{~A}$. The rapid change in the current dependence indicates the saturation of the ferromagnetic materials. It should be noted that the measured $\mathrm{AC}$ losses per cycle increases with the operating frequency, which is possibly due to increased eddy current loss in normal metal parts of the superconducting wire at higher operating frequency.

\section{Solenoid coil:}

$\mathrm{AC}$ losses of the coil were tested when the critical current was measured as $34 \mathrm{~A}$ and $47 \mathrm{~A}$, respectively. The temperature measurement of the coil was not accurate as the temperature sensor cannot be fixed on the G10 tube perfectly. AC losses were measured with supply frequency of $16 \mathrm{~Hz}, 32 \mathrm{~Hz}$ and $64 \mathrm{~Hz}$, and presented in Fig. 6. Again the measured AC losses with different critical currents are similar, which means the $\mathrm{AC}$ losses are independent of operating temperature.

\section{SimUlation RESUltS AND Discussion}

The hysteresis loss in $\mathrm{MgB}_{2}$ superconductor and eddy current loss in normal metal parts of the superconducting wire for the straight wire estimated by FE model using COMSOL Multiphysics software are presented in this section.

\section{A. Hysteresis Loss}

A two dimensional (2D) FE model was built to predict the hysteresis loss in $\mathrm{MgB}_{2}$ superconductor. $\mathrm{MgB}_{2}$ filaments were modeled as round filaments with iron barrier for simplification. $\mathbf{H}$-formulation was applied in this model to calculate current density distribution $\mathbf{J}$ and electric field distribution $\mathbf{E}$ [18], [19]. 


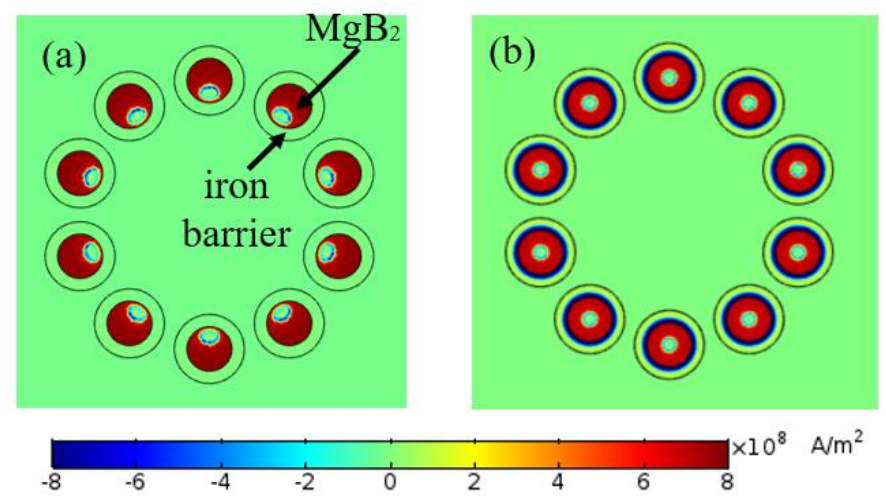

Fig. 7. Current density distribution at peak current of 250 A: (a) relative permeability of iron barrier is 1 and (b) relative permeability of iron barrier is 1000 .

Two relative permeability $\mu_{\mathrm{r}}$ values of iron barrier were considered: 1 for non-magnetic material and 1000 for ferromagnetic material. The current density distributions with two different relative permeability when the peak transport current is $250 \mathrm{~A}$ are illustrated in Fig. 7 (a) and (b), respectively. As can be seen in Fig. 7 (a), the current penetrates from the outside of the entire wire and there are clear interactions between 10 filaments. However, as shown in Fig. 7 (b), the reverse current is induced in magnetic iron barrier and the 10 filaments seem isolated from each other.

Hysteresis loss can be calculated as:

$$
Q=\int_{0}^{T} \int_{S} E \times J d s d t
$$

where $E$ is the electric field strength, $J$ is the current density, and $S$ denotes the area of superconducting material.

Fig. 8 shows the hysteresis loss in $\mathrm{MgB}_{2}$ superconductor predicted by analytical Norris model and finite element simulation as a function of peak transport current on a logarithmic scale.

Two conditions were considered using Norris model: 10 filaments were considered to be isolated from each other and the total hysteresis loss is 10 times that of single filament; 10 filaments were considered as a single big filament which has the same cross-sectional area as 10 filaments. The hysteresis loss of the first condition is one tenth of the second condition. The hysteresis loss for the multifilamentary $\mathrm{MgB}_{2}$ wire should fall between these two extreme conditions.

When the relative permeability of the iron barrier is 1 , it is clear that the hysteresis loss falls between two condition predicted by Norris model, which proves that the $\mathrm{MgB}_{2}$ filaments interact with each other. When the relative permeability of the iron barrier is 1000 , the hysteresis loss is more close to the condition that 10 filaments isolated from each other. This means the ferromagnetic barrier has a shielding effect on superconductor filament.

The trend of analytical and numerical results is consistent, the hysteresis loss of this wire is proportional to the cube of

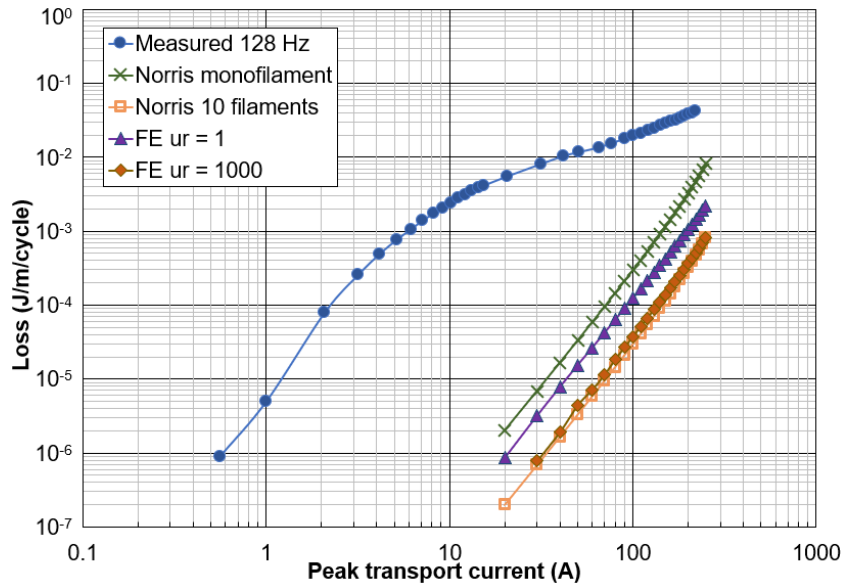

Fig. 8. Analytical and FE simulation results of hysteresis loss in $\mathrm{MgB}_{2}$ superconductor at $23 \mathrm{~K}$.

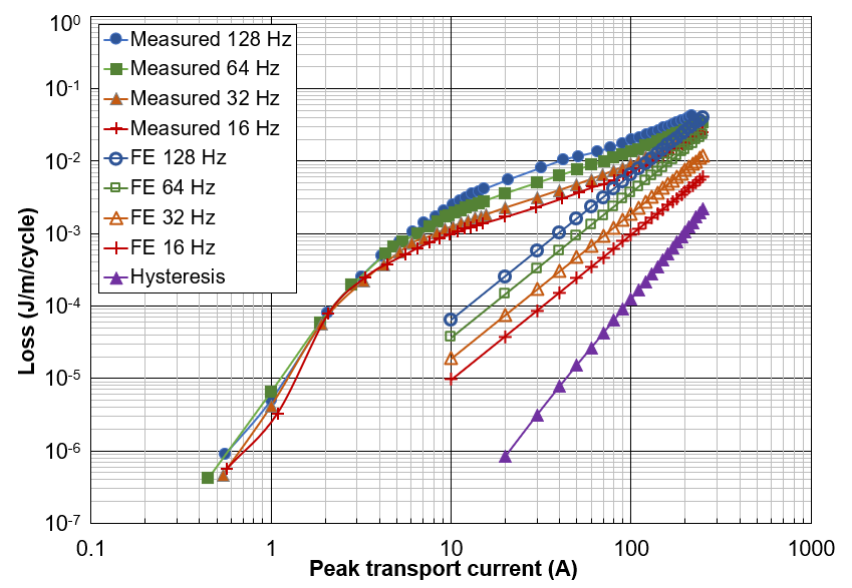

Fig. 9. Measured AC losses, hysteresis losses and eddy current losses (FE model) in $\mathrm{MgB}_{2}$ straight wire at $23 \mathrm{~K}$.

transport current. However, compared with measured AC losses, hysteresis loss only accounts for a small fraction of total AC losses. This explains why the AC loss is independent of operating temperature although hysteresis loss changes with operating temperature.

\section{B. Eddy Current Loss}

Eddy current loss in copper matrix, iron barrier, and Monel sheath was estimated using magnetic vector potential $\mathbf{A}$ as a variable [20]. As the ferromagnetic iron barrier would be saturated when the current is higher than $5 \mathrm{~A}$, the relative permeability of iron barrier was set as 1 in this model.

Fig. 9 presents measured AC losses, estimated hysteresis loss and estimated eddy current loss as a function of peak transport current. Eddy current loss per cycle increases with operating frequency, and it is proportional to the square of transport current. The proportion of eddy current loss in normal metal parts of the superconducting wire grows with transport current. At the operating frequency of $64 \mathrm{~Hz}$ and 128 $\mathrm{Hz}$, eddy current loss dominates AC losses when the transport current is close to the critical current. 
TABLE I

EDDY CURRENT LOSS IN DIFFERENT CONDUCTORS WHEN PEAK TRANSPORT CURRENT IS $250 \mathrm{~A}$

\begin{tabular}{ccccc}
\hline \hline $\begin{array}{c}\text { Frequency } \\
(\mathrm{Hz})\end{array}$ & $\begin{array}{c}\mathrm{Cu} \\
(\mathrm{J} / \mathrm{m} / \text { cycle })\end{array}$ & $\begin{array}{c}\mathrm{Fe} \\
(\mathrm{J} / \mathrm{m} / \text { cycle })\end{array}$ & $\begin{array}{c}\text { Monel } \\
(\mathrm{J} / \mathrm{m} / \text { cycle })\end{array}$ & $\begin{array}{c}\text { Total } \\
(\mathrm{J} / \mathrm{m} / \text { cycle })\end{array}$ \\
\hline 16 & $3.10 \times 10^{-3}$ & $2.87 \times 10^{-3}$ & $5.60 \times 10^{-6}$ & $5.98 \times 10^{-3}$ \\
32 & $6.15 \times 10^{-3}$ & $5.68 \times 10^{-3}$ & $1.11 \times 10^{-5}$ & $1.18 \times 10^{-2}$ \\
64 & $1.19 \times 10^{-2}$ & $1.10 \times 10^{-2}$ & $2.14 \times 10^{-5}$ & $2.29 \times 10^{-2}$ \\
128 & $2.08 \times 10^{-2}$ & $1.92 \times 10^{-2}$ & $3.76 \times 10^{-5}$ & $4.01 \times 10^{-2}$ \\
\hline
\end{tabular}

Table I presents eddy current loss in copper matrix, iron barrier, and Monel sheath. Eddy current loss is dominated by copper matrix and iron barrier whilst the loss in Monel sheath can be ignored. It can also be seen that eddy current loss increases with operating frequency squared, which explains why measured $\mathrm{AC}$ losses increase as the operating frequency increases.

Ferromagnetic hysteresis loss contributes to a large proportion of AC losses, in particular when the transport current is low. Hence, the use of ferromagnetic materials should be minimized to reduce AC losses.

\section{CONCLUSION}

The measured $\mathrm{AC}$ losses of Hitachi $\mathrm{MgB}_{2}$ wire are independent of operating temperature. Hysteresis loss in superconducting material accounts for a small fraction of AC losses. It should be pointed out that hysteresis loss cannot be estimated precisely in this case due to strong magnetic shielding by iron barriers and dominating losses in magnetic materials together with eddy current loss in copper.

AC losses per cycle increase with the operating frequency, which is due to increased eddy current loss in the normal metal parts of the superconducting wire. Eddy current loss dominates AC losses when the transport current is close to the critical current at operating frequency of $64 \mathrm{~Hz}$ and $128 \mathrm{~Hz}$. And eddy current loss can be reduced by using high resistivity material such as GlidCop and stainless steel.

The ferromagnetic barrier has a shielding effect on superconductor filaments, which reduces superconductor hysteresis loss, but produces higher ferromagnetic hysteresis loss. The use of ferromagnetic materials should be minimized to reduce AC losses.

\section{ACKNOWLEDGMENT}

The authors would like to thank Hitachi Ltd., for providing the $\mathrm{MgB}_{2}$ samples.

\section{REFERENCES}

[1] F. Grilli, E. Pardo, A. Stenvall, D. N. Nguyen, W. Yuan, and F. Gömöry, "Computation of losses in HTS under the action of varying magnetic fields and currents," IEEE Transactions on Applied Superconductivity, vol. 24, no. 1, pp. 78-110, 2014.

[2] L. Rostila et al., "Magnesium diboride wires with nonmagnetic matrices-AC loss measurements and numerical calculations," IEEE
Transactions on Applied Superconductivity, vol. 21, no. 3, pp. 33383341, 2011

[3] J. Kováč, J. Šouc, P. Kováč, and I. Hušek, "AC losses of single-core $\mathrm{MgB}_{2}$ wires with different metallic sheaths," Physica C: Superconductivity and its Applications, vol. 519, pp. 95-99, 2015.

[4] S. Safran, J. Šouc, F. Gömöry, P. Kovac, and A. Gencer, "Experimentally determined magnetization ac losses of mono and multifilamentary $\mathrm{MgB}_{2}$ wires," Journal of superconductivity and novel magnetism, vol. 26, no. 5, pp. 1557-1561, 2013.

[5] J. Kováč, J. Šouc, P. Kováč, and I. Hušek, "Magnetization AC losses in $\mathrm{MgB}_{2}$ wires made by IMD process," Superconductor Science and Technology, vol. 28, no. 1, p. 015013, 2014.

[6] S. S. Kalsi, Applications of high temperature superconductors to electric power equipment: John Wiley \& Sons, 2011.

[7] J. Kováč, J. Šouc, P. Kováč, I. Hušek, and F. Gömöry, "Experimental study of magnetization $\mathrm{AC}$ loss in $\mathrm{MgB}_{2}$ wires and cables with nonmagnetic sheath," Physica C: Superconductivity, vol. 495, pp. 182-186, 2013

[8] J. Nagamatsu, N. Nakagawa, T. Muranaka, Y. Zenitani, and J. Akimitsu, "Superconductivity at $39 \mathrm{~K}$ in magnesium diboride," Nature, vol. 410, no. 6824, pp. 63, 2001.

[9] P. Kováč, I. Hušek, J. Kováč, T. Melišek, M. Kulich, and L. Kopera, "Filamentary $\mathrm{MgB}_{2}$ wires with low magnetization AC losses," IEEE Transactions on Applied Superconductivity, vol. 26, no. 6, pp. 1-5, 2016

[10] M. Polák et al., "AC losses and transverse resistivity in filamentary $\mathrm{MgB}_{2}$ tape with Ti barriers," Physica C: Superconductivity, vol. 471, no. 13-14, pp. 389-394, 2011.

[11] K. Kajikawa et al., "Dependence of transport-current losses in $\mathrm{MgB}_{2}$ superconducting wire on temperature and frequency," IEEE Transactions on Applied Superconductivity, vol. 20, no. 3, p. 2111, 2010.

[12] Z. Hong, L. Ye, M. Majoros, A. Campbell, and T. Coombs, "Numerical estimation of $\mathrm{AC}$ loss in $\mathrm{MgB}_{2}$ wires in self-field condition," Journal of Superconductivity and Novel Magnetism, vol. 21, no. 3, pp. 205-211, 2008

[13] E. Young, M. Bianchetti, G. Grasso, and Y. Yang, "Characteristics of $\mathrm{AC}$ Loss in multifilamentary $\mathrm{MgB}_{2}$ tapes," IEEE Transactions on Applied Superconductivity, vol. 17, no. 2, pp. 2945-2948, 2007.

[14] W. Norris, "Calculation of hysteresis losses in hard superconductors carrying AC: isolated conductors and edges of thin sheets," Journal of Physics D: Applied Physics, vol. 3, no. 4, pp. 489, 1970.

[15] H. Tanaka, M. Kodama, Y. Ichiki, T. Kusunoki, H. Kotaki, T. Suzuki, $\mathrm{K}$. Nishi, and K. Okamoto, "Conduction-cooled $\mathrm{MgB}_{2}$ coil in maximum self-magnetic flux density $2.3 \mathrm{~T}$ made with 300 -meter-long multifilamentary $\mathrm{MgB}_{2}$ wire," IEEE Transactions on Applied Superconductivity, vol. 27, no. 4, pp. 1-4, 2017.

[16] H. Tanaka, T. Suzuki, M. Kodama, Y. Ichiki, T. Haba, and K. Okamoto, "Tensile and bending stress tolerance on round $\mathrm{MgB}_{2}$ wire made by in situ PIT process," IEEE Transactions on Applied Superconductivity, vol. 28 , no. 4 , pp. $1-5,2018$.

[17] X. Pei, A. C. Smith, and M. Barnes, "AC losses measurement and analysis for a $2 \mathrm{G}$ YBCO coil in metallic containment vessels," IEEE Transactions on Applied Superconductivity, vol. 27, no. 4, pp. 1-5, 2017.

[18] Z. Hong, "Numerical solution of critical state in superconductivity by finite element method," Doctor of Philosophy Thesis, University of Cambridge, 2008

[19] Y. Wang, H. Song, W. Yuan, Z. Jin, and Z. Hong, "Ramping turn-toturn loss and magnetization loss of a No-Insulation (RE) Ba2Cu3Ox high temperature superconductor pancake coil," Journal of Applied Physics, vol. 121, no. 11, Mar 2017, Art. no. 113903.

[20] COMSOL Multiphysics. "Models.acdc.eddy_currents." [Online]. Available: https://uk.comsol.com/model/eddy-currents-3d-970, Accessed on: Sept. 10, 2018 